\title{
The Large-Time Behavior of the Scalar, Genuinely Nonlinear Lax-Friedrichs Scheme*
}

\author{
By Eitan Tadmor**
}

\begin{abstract}
We study the Lax-Friedrichs scheme, approximating the scalar, genuinely nonlinear conservation law $u_{t}+f_{x}(u)=0$, where $f(u)$ is, say, strictly convex, $\ddot{f} \geqslant \dot{a}_{*}>0$. We show that the divided differences of the numerical solution at time $t$ do not exceed $2\left(t_{a_{*}}\right)^{-1}$. This one-sided Lipschitz boundedness is in complete agreement with the corresponding estimate one has in the differential case; in particular, it is independent of the initial amplitude, in sharp contrast to linear problems. It guarantees the entropy compactness of the scheme in this case, as well as providing a quantitative insight into the large-time behavior of the numerical computation.
\end{abstract}

Introduction. We consider monotonicity-preserving schemes of the 3-point conservative form

$$
v_{\nu}(t+k)=v_{\nu}(t)-\lambda\left[h\left(v_{\nu}(t), v_{\nu+1}(t)\right)-h\left(v_{\nu-1}(t), v_{\nu}(t)\right)\right],
$$

serving as consistent approximations to the scalar conservation law

$$
\frac{\partial u}{\partial t}(x, t)+\frac{\partial f}{\partial x}(u(x, t))=0
$$

and subject to initial data

$$
\left.v_{\nu}(t)\right|_{t=0}=u\left(x_{\nu}, 0\right), \quad u(x, 0) \in L^{1} \cap L^{\infty} \cap B V .
$$

Here, $v_{\nu}(t) \equiv v\left(x_{\nu}, t\right)$ denotes the approximation value at the gridpoint $\left(x_{\nu} \equiv \nu \Delta x, t\right)$, $k$ and $\Delta x$ are, respectively, the time-step and mesh size such that the mesh ratio $\lambda \equiv k / \Delta x$ is kept fixed, and $h(\cdot, \cdot)$ is the Lipschitz continuous numerical flux consistent with the differential one, $h(v, v)=f(v)$.

Studying conservative difference approximations to (1.2), one aims at having

(i) compactness,

(ii) entropy condition.

By compactness we merely mean the compactness of the family of solutions

$$
\{v(\cdot, t) \equiv v(\cdot, t ; \Delta x), 0 \leqslant t \leqslant T, 0 \leqslant \Delta x \leqslant \varepsilon\} .
$$

A standard tool used in that direction, e.g., [1], [3], [6], [11], is to guarantee that the total variation $\operatorname{TV}[v(t)] \equiv \sum_{\nu}\left|v_{\nu+1}(t)-v_{\nu}(t)\right|$ remains bounded in time, $v \in$ $L^{\infty}(B V,[0, T])$ : since the mean value $\bar{v}(t)=\sum_{\nu} v_{\nu}(t) \Delta x$ is independent of $t$, it then

Received May 26, 1983.

1980 Mathematics Subject Classification. Primary 65P05, 35L65

* Research was supported by the National Aeronautics and Space Administration under NASA Contract No. NAS1-17070 while the author was in residence at ICASE, NASA Langley Research Center, Hampton, VA 23665.

${ }^{* *}$ Current Address: School of Mathematical Sciences, Tel-Aviv University, Tel-Aviv 69978, Israel. 
follows that $v \in L^{\infty}\left(L^{1} \cap L^{\infty},[0, T]\right)$; a classical argument which involves Helly's theorem, the diagonal process and Lipschitz continuity of $|v(\cdot, t)|_{L^{1}}$, implies $L_{\text {loc }}^{1}$ compactness. By compactness there follows the existence of a subsequence $L^{1}$-limit solution $v(x, t), v(x, t)=\lim _{x=\nu \Delta x^{\prime}, \Delta x^{\prime} \rightarrow 0} v_{\nu}\left(t ; \Delta x^{\prime}\right), 0 \leqslant t \leqslant T$, satisfying (1.2) in the weak sense. In Section 2 we show that 3-point monotonicity-preserving schemes are exactly those whose total variation does not increase in time; in particular, therefore, they admit a limit-weak solution.

It is well-known that independently of the initial smoothness, weak solutions of (1.2) are, in general, not unique. By the entropy condition, we refer to a variety of criteria which single out the so-called physically relevant solution, thus guarantee uniqueness: geometrically they require characteristics to propagate toward shocks; analytically they indicate the existence of vanishing viscosity. In the case $f$ is convex, for example, they amount to Oleinik's (E) condition requiring $u_{\text {left }}>u_{\text {right }}$ across shock discontinuities. A standard way used to verify the entropy condition, e.g., [1], [7], [13], is by constructing a discrete entropy pair satisfying an entropy inequality. Unfortunately, the limit solutions of monotonicity-preserving schemes are not necessarily the physically relevant ones-examples of limit solutions violating the entropy condition in this case are well known (e.g., Example 2.4 below).

Monotone schemes - those for which the RHS of (1.1) is nondecreasing in each of its $v$-arguments - is by now a classical example for a subclass of monotonicity preserving schemes, capturing both the compactness and the entropy requirements. This has been shown by successfully implementing ideas along the above lines, e.g. [1], [7], [13]. Recently, Osher [13] introduced, for the method of lines, a general E class of monotonicity-preserving schemes enjoying both properties of entropysatisfying compactness.

Identifying 3-point conservative schemes according to their numerical viscosity coefficient [6], we arrive, in Section 2, to the following concise characterizations: while monotonicity-preserving schemes (compactness) are exactly those having numerical dissipation no more than Lax-Friedrichs (LF) scheme, no less than Murman's scheme, entropy-satisfying schemes are those further restricted by having no less dissipation than Godunov's scheme; the latter is, in fact, the fully discrete analogue of Osher's E schemes.

In both cases, the LF scheme plays a special role as the one having the most allowable numerical dissipation. In Section 3 we begin studying the LF scheme in the genuinely nonlinear case where $f$ is, say, strictly convex, $\dot{a}_{*}=\operatorname{Min} \ddot{f}(v)>0$. (Here and elsewhere in the paper, ' denotes differentiation and $a(v)$ stands for $\dot{f}(v)$.) We show that the following one-sided Lipschitz condition holds

$$
\sup _{\nu} \frac{v_{\nu+1}(t)-v_{\nu-1}(t)}{2 \Delta x} \leqslant \frac{2}{t \dot{a}_{*}} \text {. }
$$

The one-sided Lipschitz bound is in complete agreement with the corresponding estimate one has in the differential case [10, Theorem 3.1]. In particular, it is independent of the initial amplitude; this is in sharp contrast to the situation in the linear problem. Estimate (L) guarantees both compactness and the entropy condition as well as providing quantitative insight into the behavior of the numerical 
solution. In Section 4 we verify the (L) estimate for the LF scheme when the CFL condition is replaced by the weaker monotonicity-preserving requirement.

To motivate (L), one differentiates (1.2) to find that along any characteristic $d x / d t=a(u(x, t))$ we have $d\left(u_{x}\right) / d t=-\dot{a}\left(u_{x}\right)^{2}$; the latter equation is dominated by the characteristic ODE

$$
\frac{d w}{d t}=-\dot{a}_{*} w^{2}, \quad w=w(t)
$$

For the last ODE one has $w(t) \leqslant 1 / t \dot{a}_{*}$; since physically relevant solutions of (1.2) are exactly those whose characteristics can be drawn backward to the initial line $t=0$, we conclude that $u_{x} \leqslant 1 / t \dot{a}_{*}$ (weakly), which is the differential analogue of having (L). Interestingly, the very same equation which rules out the existence of (long-time) strong solutions, e.g., [8], [9], can be used to show the existence of a physically relevant weak one. (Alternatively, the following simpler geometric argument holds: the straight characteristics issued backward from $\left(x_{1}, t\right)$ and $\left(x_{2}, t\right)$ meet the initial line $t=0$, at $x_{1}-t a\left(u\left(x_{2}, t\right)\right)$ and $x_{2}-t a\left(u\left(x_{2}, t\right)\right)$, respectively; the requirement for these characteristics not to intersect yields, after little rearrangement,

$$
\left.\frac{a\left(u\left(x_{2}, t\right)\right)-a\left(u\left(x_{1}, t\right)\right)}{x_{2}-x_{1}} \leqslant \frac{1}{t}, \quad x_{2}>x_{1} .\right)
$$

We close by saying that most likely the one-sided (L) condition holds for other schemes-those in the $E$ class are, of course, natural candidates. If so, (L) will provide, in the genuinely nonlinear case, a unified alternative to the standard total variation boundedness/entropy inequality approach, in showing the entropy-satisfying compactness.

2. Three-Point Monotonicity-Preserving Schemes. We start by considering 3-point schemes in an increment form

$$
v_{\nu}(t+k)=v_{\nu}(t)+C_{\nu+1 / 2}^{+} \Delta v_{\nu}-C_{\nu-1 / 2}^{-} \Delta v_{\nu-1},
$$

where

$$
C_{\nu+1 / 2}^{ \pm} \equiv C^{ \pm}\left(v_{\nu}(t), v_{\nu+1}(t)\right)
$$

Having the scheme in such increment form is not a restriction. In fact, we have

LEMMA 2.1. Every 3-point conservative scheme (1.1) can be written in an increment form (2.1). Conversely, any 3-point incremental scheme is conservative, provided the following consistency requirement,

$$
C_{\nu+1 / 2}^{-}-C_{\nu+1 / 2}^{+} \equiv \lambda \frac{\Delta f_{\nu}}{\Delta v_{\nu}}
$$

holds. 
Proof. We follow Harten [5, Section II.3]. Suppose (2.1) admits a consistent conservative form; equating the RHS of (2.1a) and (1.1), we obtain (for simplicity, we drop the time dependence)

$$
C_{\nu+1 / 2}^{+} \Delta v_{\nu}-C_{\nu-1 / 2}^{-} \Delta v_{\nu-1}=-\lambda\left[h\left(v_{\nu}, v_{\nu+1}\right)-h\left(v_{\nu-1}, v_{\nu}\right)\right] .
$$

Setting $v_{\nu-1}=v_{\nu}$ gives

$$
C_{\nu+1 / 2}^{+} \equiv \lambda \frac{h\left(v_{\nu}, v_{\nu}\right)-h\left(v_{\nu}, v_{\nu+1}\right)}{\Delta v_{\nu}},
$$

while putting $v_{v}=v_{v+1}$ implies

$$
C_{\nu-1 / 2}^{-} \equiv \lambda \frac{h\left(v_{\nu}, v_{\nu}\right)-h\left(v_{\nu-1}, v_{\nu}\right)}{\Delta v_{\nu-1}} .
$$

Thus, the incremental coefficients are necessarily those given in (2.3). We note that, using (2.3), the consistency condition $h(v, v)=f(v)$ amounts to having (2.2).

Now, suppose (2.2) holds, and define the consistent numerical flux

$$
h\left(v_{\nu}, v_{\nu+1}\right) \equiv f\left(v_{\nu}\right)-\frac{1}{\lambda} C_{\nu+1 / 2}^{+} \Delta v_{\nu} .
$$

Making use of (2.2), we find

$$
\left(2.4 \mathrm{~b}_{:-1}\right) \quad h\left(v_{\nu-1}, v_{\nu}\right)=f\left(v_{\nu-1}\right)-\frac{1}{\lambda} C_{\nu-1 / 2}^{+} \Delta v_{\nu-1}=f\left(v_{\nu}\right)-\frac{1}{\lambda} C_{\nu-1 / 2}^{-} \Delta v_{\nu-1} \text {, }
$$

which puts the RHS of (2.1a) in the consistent conservative form (1.1), thus completing the proof.

An essential property characterizing the scalar differential solution operator, which is highly desirable to be retained in the discrete framework as well, is preserving monotone profiles. We have

LEMMA 2.2. Three-point monotonicity-preserving schemes are exactly those whose total variation is nonincreasing. They are characterized by the set of inequalities

$$
C_{\nu+1 / 2}^{+} \geqslant 0, \quad C_{\nu+1 / 2}^{-} \geqslant 0, \quad 1-C_{\nu+1 / 2}^{+}-C_{\nu+1 / 2}^{-} \geqslant 0 .
$$

Proof. Differencing (2.1a), we obtain

$$
\begin{aligned}
\Delta v_{\nu}(t+k)= & C_{\nu+3 / 2}^{+} \Delta v_{\nu+1}(t)+\left(1-C_{\nu+1 / 2}^{+}-C_{\nu+1 / 2}^{-}\right) \Delta v_{\nu}(t) \\
& +C_{\nu-1 / 2}^{-} \Delta v_{\nu-1}(t) .
\end{aligned}
$$

Setting $v_{\nu-1}(t)=v_{\nu}(t)=v_{\nu+1}(t)$, i.e., $\Delta v_{\nu-1}(t)=\Delta v_{\nu}(t)=0$, we find

$$
\Delta v_{\nu}(t+k)=C_{\nu+3 / 2}^{+} \Delta v_{\nu+1}(t) .
$$

By monotonicity preserving, the sign of $\Delta v_{\nu}(t+k)$ must agree with that of $\Delta v_{\nu+1}(t)$, and, hence, $C_{v+3 / 2}^{+} \equiv C^{+}\left(v_{v+1}, v_{v+2}\right)$ should be nonnegative for arbitrary $v_{v+1}, v_{v+2}$. The other inequalities in (2.5) follow likewise by setting $\Delta v_{\nu-1}(t)=\Delta v_{\nu+1}(t)=0$ and $\Delta v_{\nu}(t)=\Delta v_{\nu+1}(t)=0$. 
Next, we follow Harten [6] in showing that (2.4) implies the nonincrease in total variation; summing (2.6), we obtain

$$
\begin{aligned}
\operatorname{TV}[v(t+k)] \equiv & \sum_{\nu}\left|\Delta v_{\nu}(t+k)\right| \leqslant \sum_{\nu}\left|C_{\nu+3 / 2}^{+}\right|\left|\Delta v_{\nu+1}(t)\right| \\
& +\sum_{\nu}\left|\left(1-C_{\nu+1 / 2}^{+}-C_{\nu+1 / 2}^{-}\right)\right|\left|\Delta v_{\nu}(t)\right|+\sum_{\nu}\left|C_{\nu-1 / 2}^{-}\right|\left|\Delta v_{\nu-1}(t)\right| .
\end{aligned}
$$

Reindexing the first and third summations, we find, on account of (2.4), that the RHS does not exceed

$$
\begin{aligned}
\sum_{\nu} C_{\nu+1 / 2}^{+}\left|\Delta v_{\nu}(t)\right|+\sum_{\nu}\left(1-C_{\nu+1 / 2}^{+}\right. & \left.-C_{\nu+1 / 2}^{-}\right)\left|\Delta v_{\nu}(t)\right| \\
& +\sum_{\nu} C_{\nu+1 / 2}^{-}\left|\Delta v_{\nu}(t)\right|=\sum_{\nu}\left|\Delta v_{\nu}(t)\right| .
\end{aligned}
$$

Since, on the other hand, nonincreasing total variation implies monotonicity preserving [6], there follows the equivalence between the two and their characterization by (2.4).

The consistency requirement (2.2) shows that there is only one degree of freedom in setting up the recipe for a 3-point conservative scheme; let

$$
Q_{\nu+1 / 2} \equiv C_{\nu+1 / 2}^{+}+C_{\nu+1 / 2}^{-} \text {. }
$$

Then by averaging $\left(2.4 \mathrm{a}_{\nu}\right)$ and $\left(2.4 \mathrm{~b}_{\nu}\right)$ we have

$$
h\left(v_{\nu}, v_{\nu+1}\right)=\frac{1}{2}\left(f\left(v_{\nu}\right)+f\left(v_{\nu+1}\right)-\frac{1}{\lambda} Q_{\nu+1 / 2} \Delta v_{\nu}\right) .
$$

The scheme (1.1) is then recast into the form

$$
\begin{aligned}
v_{\nu}(t+k)= & v_{\nu}(t)-\frac{\lambda}{2}\left[f\left(v_{\nu+1}(t)\right)-f\left(v_{\nu-1}(t)\right)\right] \\
& +\frac{1}{2}\left[\Delta\left(Q_{\nu-1 / 2} \Delta v_{\nu-1}(t)\right)\right],
\end{aligned}
$$

which reveals the role $Q$ plays as the numerical viscosity coefficient [6]. Noting that according to (2.2)

$$
C_{\nu+1 / 2}^{ \pm} \equiv \frac{C_{\nu+1 / 2}^{+}+C_{\nu+1 / 2}^{-}}{2} \pm \frac{C_{\nu+1 / 2}^{+}-C_{\nu+1 / 2}^{-}}{2}=\frac{1}{2}\left(Q_{\nu+1 / 2} \mp \lambda \frac{\Delta f_{\nu}}{\Delta v_{\nu}}\right)
$$

we conclude

COROllary 2.3. A 3-point conservative scheme (2.9) is monotonicity preserving and total variation nonincreasing if, and only if, its numerical viscosity coefficient, $Q_{\nu+1 / 2}$, satisfies

$$
\lambda\left|\frac{\Delta f_{\nu}}{\Delta v_{\nu}}\right| \leqslant Q_{\nu+1 / 2} \leqslant 1
$$

We turn now to considering a few examples.

Example 2.4. Murman's scheme [12] is the nonlinear conservative generalization of the upwind Courant-Isaacson-Rees (CIR) scheme

(2.12a) $v_{\nu}(t+k)=v_{\nu}(t)-\lambda\left[s_{\nu+1 / 2}\left(f_{\nu+1}-f_{\nu}\right)+\left(1-s_{\nu-1 / 2}\right)\left(f_{\nu}-f_{\nu-1}\right)\right]$, 
where

$$
s_{\nu+1 / 2}= \begin{cases}1, & \frac{\Delta f_{\nu}}{\Delta v_{\nu}}<0 \\ 0, & \frac{\Delta f_{\nu}}{\Delta v_{\nu}}>0\end{cases}
$$

Noting that $s_{\nu+1 / 2}=\frac{1}{2}\left(1-\operatorname{sign}\left[\Delta f_{\nu} / \Delta v_{\nu}\right]\right)$, its incremental form (2.1a) amounts to

$$
C_{\nu+1 / 2}^{ \pm}=\frac{\lambda}{2}\left(\operatorname{sign}\left[\frac{\Delta f_{\nu}}{\Delta v_{\nu}}\right] \mp 1\right) \frac{\Delta f_{\nu}}{\Delta v_{\nu}}=\frac{\lambda}{2}\left(\left|\frac{\Delta f_{\nu}}{\Delta v_{\nu}}\right| \mp \frac{\Delta f_{\nu}}{\Delta v_{\nu}}\right),
$$

and it admits the consistent conservative form (1.1) with

$$
h\left(v_{\nu}, v_{\nu+1}\right)=\frac{1}{2}\left(f_{\nu}+f_{\nu+1}-\left|\frac{\Delta f_{\nu}}{\Delta v_{\nu}}\right| \Delta v_{\nu}\right) .
$$

Its numerical viscosity coefficient is given by

$$
Q_{\mathrm{CIR}, \nu+1 / 2}=\lambda\left|\frac{\Delta f_{\nu}}{\Delta v_{\nu}}\right|,
$$

and hence it meets the monotonicity-preserving requirement $(2.11)$ provided the CFL-like condition $\lambda \sup _{\nu}\left|\Delta f_{\nu} / \Delta v_{\nu}\right| \leqslant 1$ is fulfilled. If this is the case, then by Lemma 2.2 and the previously argued compactness, the scheme admits a limit solution $v(x, t)=\lim _{x=\nu \Delta x^{\prime}, \Delta x^{\prime} \rightarrow 0} v_{\nu}\left(t ; \Delta x^{\prime}\right)$. It is well known that this limit solution nevertheless may turn out to be a physically irrelevant one as shown, for example, by choosing initial data $v_{\nu}(t=0)=\operatorname{sign}(\nu-1 / 2)$ : with $f(u)=u^{2}$, the scheme admits this initial discontinuity as an "expansive shock" steady-state solution rather than dissolving it as a rarefaction, the reason being its lacking of (i.e., zero) dissipation in this case.

Example 2.5. Godunov's scheme [4] is determined by the numerical flux

$$
h\left(v_{\nu}, v_{\nu+1}\right)=f\left(u_{R}\left(x_{\nu+1 / 2}, k\right)\right) \text {, }
$$

where $u_{R}(x, t)$ is the solution of the Riemann problem (1.1) with initial data

$$
u_{R}(x, t=0)= \begin{cases}v_{\nu}, & x \leqslant x_{\nu+1 / 2} \\ v_{\nu+1}, & x>x_{\nu+1 / 2}\end{cases}
$$

Consider, for simplicity, the convex case, $\ddot{f}>0$. Except for the sonic rarefaction case, $u_{R}\left(x_{\nu+1 / 2}, k\right)$ takes the value of either $v_{\nu+1}$ or $v_{\nu}$; by (2.8), $\lambda^{-1} Q_{\nu+1 / 2} \Delta v_{\nu}$ equals the difference between the sum $f_{\nu}+f_{\nu+1}$ and twice the numerical flux $h\left(v_{\nu}, v_{\nu+1}\right)$. Hence the numerical viscosity coefficient in this case is given by

$$
Q_{G, \nu+1 / 2}= \begin{cases}\lambda \frac{f_{\nu+1}-2 f(\mathbf{v})+f_{\nu}}{\Delta v_{\nu}} & \text { if } a\left(v_{\nu}\right)<a(\mathbf{v})=0<a\left(v_{\nu+1}\right), \\ \lambda\left|\frac{\Delta f_{\nu}}{\Delta v_{\nu}}\right| & \text { otherwise. }\end{cases}
$$

We remark that, in the general nonconvex case, one has, due to Osher [13, Section 2],

$$
h_{G}\left(v_{\nu}, v_{\nu+1}\right)=\operatorname{Min}_{v_{\nu+1 / 2} \leqslant v \leqslant v_{\nu+1 / 2}^{\max }}\left[\operatorname{sign}\left(v_{\nu+1}-v_{\nu}\right) f(v)\right]
$$


where $v_{\nu+1 / 2}^{\min } / \max =\operatorname{Min} / \operatorname{Max}\left(v_{\nu}, v_{\nu+1}\right)$. Hence, the numerical viscosity coefficient of Godunov's scheme boils down to $\lambda$ times

i.e.,

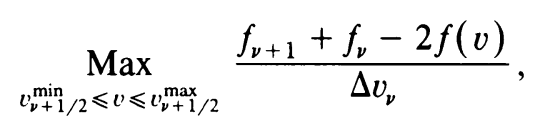

$$
Q_{G, \nu+1 / 2}= \begin{cases}\lambda \operatorname{Max} \frac{f_{\nu+1}+f_{\nu}-2 f(v)}{\Delta v_{\nu}} & \text { if } a(v) \text { vanishes, } v_{\nu+1 / 2}^{\min } \leqslant v \leqslant v_{\nu+1 / 2}^{\max }, \\ \lambda\left|\frac{\Delta f_{\nu}}{\Delta v_{\nu}}\right| & \text { if } a(v) \neq 0, v_{\nu+1 / 2}^{\min } \leqslant v \leqslant v_{\nu+1 / 2}^{\max .}\end{cases}
$$

Thus, Godunov deviates from the CIR scheme exactly where the latter fails-it introduces nonzero dissipation in the sonic rarefaction case.

Example 2.6. The Engquist-Osher (EO) scheme [2] has numerical flux

$$
h\left(v_{\nu}, v_{\nu+1}\right)=\frac{1}{2}\left(f_{\nu}+f_{\nu+1}-\int_{v_{\nu}}^{v_{\nu+1}}|\dot{f}(v)| d v\right),
$$

and hence its numerical dissipation coefficient in the convex case $\ddot{f}>0$ is given by

$$
\begin{aligned}
Q_{\mathrm{EO}, \nu+1 / 2} & =\lambda \frac{1}{\Delta v_{\nu}} \int_{v_{\nu}}^{v_{\nu+1}}|\dot{f}(v)| d v \\
& = \begin{cases}\lambda \frac{f_{\nu+1}-2 f(\mathbf{v})+f_{\nu}}{\left|\Delta v_{\nu}\right|} & \text { if } a\left(v_{\nu}\right) \cdot a\left(v_{\nu+1}\right)<0, \\
\lambda\left|\frac{\Delta f_{\nu}}{\Delta v_{\nu}}\right| & \text { otherwise. }\end{cases}
\end{aligned}
$$

Thus, it deviates from the CIR scheme in the sonic case, introducing even more dissipation than Godunov does in the sonic shock case-in fact, it treats the latter case, $a\left(v_{\nu}\right)>0>a\left(v_{\nu+1}\right)$, as a "compressive rarefaction."

Example 2.7. The Lax-Friedrichs (LF) scheme is given by

$$
v_{\nu}(t+k)=\frac{1}{2}\left(v_{\nu-1}(t)+v_{\nu+1}(t)\right)-\frac{\lambda}{2}\left(f \left(v_{\nu+1}(t)-f\left(v_{\nu-1}(t)\right)\right.\right.
$$

Its incremental coefficients, numerical flux and numerical viscosity coefficient are given respectively by

$$
\begin{gathered}
C_{\nu+1 / 2}^{ \pm}=\frac{1}{2}\left(1 \mp \lambda \frac{\Delta f_{\nu}}{\Delta f_{\nu}}\right), \\
h\left(v_{\nu}, v_{\nu+1}\right)=\frac{1}{2}\left(f_{\nu}+f_{\nu+1}-\frac{1}{\lambda} \Delta v_{\nu}\right), \\
Q_{\mathrm{LF}, \nu+1 / 2} \equiv 1 .
\end{gathered}
$$

Thus, the LF scheme is monotonicity-preserving provided the CFL-like condition $\lambda \sup _{\nu}\left|\Delta f_{\nu} / \Delta v_{\nu}\right| \leqslant 1$ is met, and hence admitting a limit solution in this case; interestingly, as we shall see later on, this solution still may be the physically irrelevant one. Only upon strengthening the CFL condition, $\lambda \sup _{v}|a(v)| \leqslant 1$, will we get the desired convergence due to the scheme monotonicity, as is the case with Godunov and EO schemes. 
In view of the above examples, we see that the CIR and LF schemes have, respectively, the least and the most numerical dissipation allowed under the monotonicity-preserving requirement, as (2.11), (2.12d), and (2.15d) read

COROLlary 2.8. A 3-point scheme is monotonicity-preserving if, and only if, its numerical viscosity coefficient, $Q_{\nu+1 / 2}$, satisfies

$$
Q_{\mathrm{CIR}, \nu+1 / 2} \leqslant Q_{\nu+1 / 2} \leqslant Q_{\mathrm{LF}, \nu+1 / 2} .
$$

In [13], Osher introduces, for the method of lines, a class of $E$ schemes which according to our terminology can be interpreted as exactly those having no less numerical dissipation than Godunov's; Osher showed that such E schemes satisfy the entropy condition. Carrying on his ideas to the fully discrete case we formulate, though do not prove,

COROLlARY 2.9. A 3-point scheme is monotonicity-preserving (compactness) and entropy satisfying if its numerical viscosity coefficient is further restricted by

$$
Q_{G, \nu+1 / 2} \leqslant Q_{\nu+1 / 2} \leqslant Q_{\mathrm{LF}, \nu+1 / 2} .
$$

Finally, we note that all the above-mentioned schemes are first order accurate. This is not a coincidence, since generically we have

LEMMA 2.10. Any 3-point monotonicity-preserving scheme is of first order accuracy.

Proof. The truncation error for a smooth solution $u$ of (1.2a) is given, modulo third order terms, by

$$
-\frac{(\Delta t)^{2}}{2}\left[\left\{Q(u, u)-\lambda^{2} a^{2}(u)\right\} u_{x}\right]_{x}
$$

By Corollary $2.3, \lambda|a(u)| \leqslant Q(u, u) \leqslant 1$, which implies that the coefficient inside the inner curly brackets is nonnegative

$$
Q(u, u)-\lambda^{2} a^{2}(u) \geqslant \lambda|a(u)| \cdot(1-\lambda|a(u)|) \geqslant 0 ;
$$

indeed, unless $\lambda\left|\Delta f_{\nu} / \Delta v_{\nu}\right| \equiv 1$ where the scheme is reduced to the trivial nongeneric case of pure translation, strict inequalities hold, excluding more than first order accuracy. We remark that an alternative proof can be given by the same argument used to show first order accuracy for monotone schemes: the first two inequalities which characterize monotonicity-preserving in (2.5) express, according to (2.3), the fact that upon setting $v=v_{\nu-1}=v_{\nu}=v_{\nu+1}$, the RHS of (1.1), $H\left(v_{\nu-1}, v_{\nu}, v_{\nu+1}\right)$, is nondecreasing in its first and third arguments; the third inequality in (2.5) implies that the partial derivatives w.r.t. these arguments are nonnegative; noting that the dependence on the second argument is not as essential, the result follows along the lines of [7].

3. Time-Decay in the Genuinely Nonlinear LF Scheme. The Lax-Friedrichs scheme

$$
v_{\nu}(t+k)=\frac{v_{\nu+1}(t)+v_{\nu-1}(t)}{2}-\frac{\lambda}{2}\left(f_{\nu+1}-f_{\nu-1}\right)
$$

is essentially a staggered-type scheme. To simplify the notations we introduce the staggered differencing operator $\tilde{\Delta} \equiv \Delta(2 \Delta x)$ and correspondingly $\tilde{D} \equiv \tilde{\Delta} / 2 \Delta x$; $\tilde{D}_{\nu}(t)$ abbreviates $\tilde{D} v_{\nu}(t), \tilde{D}(t)=\sup _{\nu} \tilde{D}_{\nu}(t)$. 
We are interested in the time-decay of the numerical solution $v_{\nu}(t)$ in the genuinely nonlinear case, i.e., when $\dot{a}(v) \neq 0$ for all $v$, say when $f(v)$ is strictly convex

$$
\dot{a}_{*} \equiv \underset{v}{\operatorname{Min}} \dot{a}(v)>0
$$

THEOREM 3.1. Consider the LF scheme (3.1) under the CFL condition

$$
\lambda \sup _{\nu}\left|a\left(v_{\nu}(t)\right)\right| \leqslant 1
$$

Then, for arbitrary $d, d>1$, we have

$$
\tilde{D}(t) \leqslant \operatorname{Max}\left[\frac{2}{(t+d k) \dot{a}_{*}}, \frac{d k}{t+d k} \cdot \tilde{D}(t=0)\right] .
$$

Proof. By induction, starting with $t=0$ where the second term inside the maximum brackets is reduced to $\tilde{D}(t=0)$. The general step follows by showing

$$
\tilde{D}(t) \leqslant \frac{d \lambda}{t+d k} M, \quad M=\operatorname{Max}\left[\frac{2}{d \lambda \dot{a}_{*}}, \Delta x \tilde{D}(t=0)\right] .
$$

Differencing (3.1) gives

$$
\tilde{\Delta} v_{\nu}(t+k)=\frac{\tilde{\Delta} v_{\nu+1}(t)+\tilde{\Delta} v_{\nu-1}(t)}{2}-\frac{\lambda}{2}\left(\tilde{\Delta} f_{\nu+1}-\tilde{\Delta} f_{\nu-1}\right) .
$$

By the strict convexity of $f$

$$
a\left(v_{\nu}\right) \tilde{\Delta} v_{\nu}+\frac{\dot{a}_{*}}{2}\left|\tilde{\Delta} v_{\nu}\right|^{2} \leqslant \tilde{\Delta} f_{\nu} \leqslant a\left(v_{\nu+2}\right) \tilde{\Delta} v_{\nu}-\frac{\dot{a}_{*}}{2}\left|\tilde{\Delta} v_{\nu}\right|^{2}
$$

The RHS inequality follows by second order Taylor expansion around $v_{\nu+2}$; the LHS, around $v_{\nu}$. Inserted into (3.5), (3.6) yields

$$
\begin{aligned}
\tilde{\Delta} v_{\nu}(t+k) \leqslant & \frac{1-\lambda a\left(v_{\nu+1}\right)}{2} \tilde{\Delta} v_{\nu+1}+\frac{1+\lambda a\left(v_{\nu+1}\right)}{2} \tilde{\Delta} v_{\nu-1} \\
& -\frac{\lambda \dot{a}_{*}}{4}\left(\left|\tilde{\Delta} v_{\nu+1}\right|^{2}+\left|\tilde{\Delta} v_{\nu-1}\right|^{2}\right)
\end{aligned}
$$

or, after division by $2 \Delta x$,

$$
\begin{aligned}
\tilde{D}_{\nu}(t+k) \leqslant & \frac{1-\lambda a\left(v_{\nu+1}\right)}{2} \tilde{D}_{\nu+1}(t)+\frac{1+\lambda a\left(v_{\nu+1}\right)}{2} \tilde{D}_{\nu-1}(t) \\
& -\frac{k \dot{a}_{*}}{2}\left(\tilde{D}_{\nu+1}^{2}(t)+\tilde{D}_{\nu-1}^{2}(t)\right) .
\end{aligned}
$$

The CFL condition implies that the sum of the first two terms on the right does not exceed $\operatorname{Max}\left[\tilde{D}_{\nu+1}(t), \tilde{D}_{\nu-1}(t)\right]$; the sum of the two terms inside the last brackets on the right is not less than $\operatorname{Max}\left[\tilde{D}_{\nu+1}(t), \tilde{D}_{\nu-1}(t)\right]^{2}$. Hence,

$$
\tilde{D}_{\nu}(t+k) \leqslant \operatorname{Max}\left[\tilde{D}_{\nu+1}(t), \tilde{D}_{\nu-1}(t)\right]-\frac{k \dot{a}_{*}}{2} \operatorname{Max}\left[\tilde{D}_{\nu+1}(t), \tilde{D}_{\nu-1}(t)\right]^{2}
$$

We distinguish between two cases:

(i) Assume $M \leqslant(t+d k) / k \dot{a}_{*} d \lambda$. The quadratic $z(1-\gamma z)$ is monotonic increasing as long as $z<1 / 2 \gamma, \gamma>0$. This is the case with the RHS of (3.8) viewed as 
such a quadratic in $z=\operatorname{Max}\left[\tilde{D}_{\nu+1}(t), \tilde{D}_{\nu-1}(t)\right]$ with $\gamma=k \dot{a}_{*} / 2$, since by assumption

$$
z=\operatorname{Max}\left[\tilde{D}_{\nu+1}(t), \tilde{D}_{\nu-1}(t)\right] \leqslant \frac{d \lambda}{t+d k} M \leqslant\left.\frac{1}{k \dot{a}_{*}} \equiv \frac{1}{2 \gamma}\right|_{\gamma=k \dot{a}_{*} / 2} .
$$

Thus increasing the RHS of (3.8) by replacing $\operatorname{Max}\left[\tilde{D}_{\nu+1}(t), \tilde{D}_{\nu-1}(t)\right]$ with its assumed upper bound $(d \lambda /(t+d k)) M$, we find

$$
\tilde{D}_{\nu}(t+k) \leqslant \frac{d \lambda}{t+d k} M\left(1-\frac{k \dot{a}_{*} d \lambda}{2(t+d k)} M\right) .
$$

Since, by definition, $M$ is greater than $2 / d \lambda \dot{a}_{*}$, the expression in parentheses does not exceed $1-k /(t+d k)$, and the last inequality yields

$$
\tilde{D}_{\nu}(t+k) \leqslant \frac{d \lambda}{t+d k} M\left(1-\frac{k}{t+d k}\right)<\frac{d \lambda}{t+k+d k} M .
$$

(ii) $M \geqslant(t+d k) / k \dot{a}_{*} d \lambda$. The quadratic $z(1-\gamma z)$ has a maximal value $1 / 4 \gamma$, $\gamma>0$. Viewing the RHS of (3.8) as such a quadratic in $z=\operatorname{Max}\left[\tilde{D}_{\nu+1}(t), \tilde{D}_{\nu-1}(t)\right]$ with $\gamma=k \dot{a}_{*} / 2$, it does not exceed a maximal value of (recall $d>1$ )

$$
\tilde{D}_{\nu}(t+k) \leqslant \frac{1}{2 k \dot{a}_{*}} \leqslant \frac{t+d k}{k \dot{a}_{*}(t+k+d k)} \equiv \frac{d \lambda}{t+k+d k} \cdot \frac{t+d k}{k \dot{a}_{*} d \lambda} \leqslant \frac{d \lambda}{t+k+d k} M .
$$

This completes the proof.

We remark that (3.2) requires verification of the CFL condition $\lambda \sup _{v}|a(v)| \leqslant 1$ only at the grid values $v=v_{\nu}(t)$. In the next section we discuss the situation when the weaker CFL-like condition $\lambda \sup _{\nu}\left|\Delta f_{\nu} / \Delta v_{\nu}\right| \leqslant 1$ due to monotonicity preserving holds. We pause here to discuss a few implications of Theorem 3.1, excluding the rarefaction-free trivial case where by $(3.7) \tilde{D}(t=0) \leqslant 0$ implies $\tilde{D}(t) \leqslant 0$ later on.

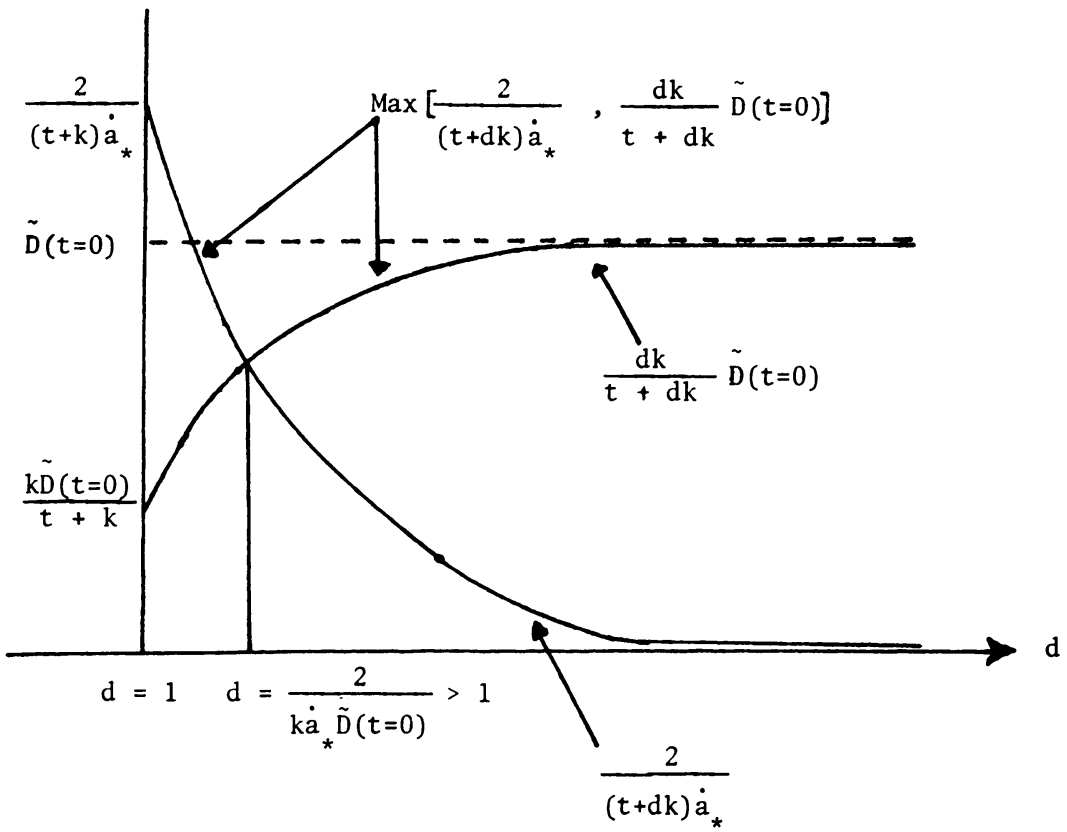

FIGURE 3-1 
The one-sided Lipschitz estimate (3.3) involves a free parameter $d, d>1$, which can be chosen so as to minimize the Lipschitz bound.

Choosing $d=2 / k \dot{a}_{*} \tilde{D}(t=0)$, see Figure 3-1, we conclude

COROLLARY 3.2. Assume the CFL condition (3.2) holds. Then, the LF scheme (3.1) satisfies

$$
\sup _{\nu} \frac{v_{\nu+1}(t)-v_{\nu-1}(t)}{2 \Delta x} \leqslant \frac{2 \tilde{D}(t=0)}{t \dot{a}_{*} \tilde{D}(t=0)+2} \leqslant \frac{2}{t \dot{a}_{*}} .
$$

Proof. We have to verify that the above choice of $d$ is admissible, i.e., that

$$
d=\frac{2}{k \dot{a}_{*} \tilde{D}(t=0)}>1 \text { or } \frac{4}{\dot{a}_{*} \tilde{\Delta} v(t=0)} \geqslant \lambda .
$$

Indeed, CFL condition (3.2) implies

$$
\lambda \dot{a}_{*} \sup _{\nu} \tilde{\Delta} v_{\nu}(t=0) \leqslant \lambda \sup _{\nu} \tilde{\Delta} a\left(v_{\nu}(t=0)\right) \leqslant 2 \lambda \sup _{\nu}\left|a\left(v_{\nu}(t=0)\right)\right|<4 .
$$

To draw global estimates from the one-sided Lipschitz condition (3.9), we have-due to the staggered nature of the LF scheme-to distinguish between the even and odd numbered grid values

$$
v_{\nu}^{\prime}(t) \equiv v_{2 v}(t), \quad v_{\nu}^{\prime \prime}(t) \equiv v_{2 v+1}(t)
$$

under the CFL assumption of Corollary 3.2, (3.9) amounts to

$$
\Delta v_{\nu}^{\prime}(t) \leqslant \frac{4 \Delta x}{t \dot{a}_{*}}, \quad \Delta v_{\nu}^{\prime \prime}(t) \leqslant \frac{4 \Delta x}{t \dot{a}_{*}} .
$$

Given a grid function $w=\left\{w_{\nu}\right\}$, its increasing (similarity decreasing) variation, $\operatorname{TV}^{+}[w]$ (similarly $\left.\operatorname{TV}^{-}[w]\right)$ is defined by

$$
\mathrm{TV}^{ \pm}[w]= \pm \sum_{ \pm \Delta w_{\nu}>0} \Delta w_{\nu}
$$

Thus, we have

$$
\operatorname{TV}[w]=\mathrm{TV}^{+}[w]+\mathrm{TV}^{-}[w]
$$

while, when restricted to the interval $\left[x_{-\infty}, x_{\infty}\right]$,

$$
\left.\mathrm{TV}^{+}[w]\right|_{\left[x_{-\infty}, x_{\infty}\right]}-\left.\mathrm{TV}^{-}[w]\right|_{\left[x_{-\infty}, x_{\infty}\right]}=\sum_{x_{\nu}=x_{-\infty}}^{x_{\infty}} \Delta w_{\nu}=w\left(x_{\infty}\right)-w\left(x_{-\infty}\right)
$$

In particular, (3.11a), (3.11b) imply

(3.11c) $\left.\operatorname{TV}[w]\right|_{\left[x_{-\infty}, x_{\infty}\right]}=\left.2 \mathrm{TV}^{ \pm}[w]\right|_{\left[x_{-\infty}, x_{\infty}\right]} \pm\left[w\left(x_{-\infty}\right)-w\left(x_{\infty}\right)\right]$.

According to this terminology, (3.10) yields

$$
\mathrm{TV}^{+}\left[v^{\prime}(t)\right] \leqslant \sum_{\Delta v_{v}^{\prime}(t)>0} \frac{4 \Delta x}{t \dot{a}_{*}} \leqslant \frac{2}{t \dot{a}_{*}} \operatorname{supp}[v(t)],
$$

and similarly for $v^{\prime \prime}(t)$.

Consider now the Cauchy problem (1.2). There are two cases:

(i) The periodic problem. Let $P$ be the period. Depending on whether it consists of an even or odd number of grid points, say even, we have by (3.12), that the 
increasing variation of $v^{\prime}(t)$ does not exceed $2 P / t \dot{a}_{*}$; because of its periodicity, (3.11c) imply that $v^{\prime}(t)$ has a total variation which equals twice its increasing one. Hence

$$
\operatorname{TV}\left[v^{\prime}(t)\right] \leqslant \frac{4 P}{t \operatorname{Min} \dot{a}\left(v^{\prime}(t)\right)} .
$$

Thus, the total variation of $v^{\prime}(t)$ per period tends to zero as $t$ tends to infinity, in an inverse linear rate, $\sim t^{-1}$. In particular, since the mean value of $v^{\prime}(t), \bar{v}^{\prime}(t)$, is conserved in time, we conclude that for $t$ large enough

$$
\sup _{\nu}\left|v_{\nu}^{\prime}(t)-\bar{v}^{\prime}(t=0)\right| \leqslant \frac{2 P}{t \dot{a}\left(\bar{v}^{\prime}(t=0)\right)} .
$$

The last two estimates are-apart from the unessential factor of two-in complete agreement with the corresponding estimates one has in the differential case [10, Theorem 4.3].

(ii) The pure Cauchy problem. Suppose the initial data $v\left(x=x_{\nu}, t=0\right)$ are supported in a finite interval of length $L$. Applying (3.11c) over the support of $v^{\prime}(t)$, we find that its total variation equals twice its increasing one; using (3.12) to bound the latter we conclude

$$
\operatorname{TV}\left[v^{\prime}(t)\right] \leqslant \frac{4}{t \dot{a}_{*}} \operatorname{supp}[v(t)]
$$

In the differential case, the solution support at time $t$ expands like $t^{1 / 2},[10$, Section 4], and (3.15) implies a total variation decay $\leqslant t^{-1 / 2}$. In the discrete case, the support of $v(t)$ does not exceed $L+2 \lambda^{-1} t$ and hence

$$
\operatorname{TV}\left[v^{\prime}(t)\right] \leqslant \frac{8}{\lambda \dot{a}_{*}}+\frac{4 L}{t \dot{a}_{*}},
$$

showing the boundedness of the total variation. We remark that in both the periodic and pure Cauchy problems, similar estimates like those derived above hold for $v^{\prime \prime}(t)$ as well, since the difference between its end values, according to the above $v^{\prime}$-estimates, is uniformly bounded. Therefore, in either case, we end up with a bound on the total variation which shows that the one-sided Lipschitz condition (3.9) guarantees the desired compactness, which in turn leads to the existence of a limit solution

$$
v(x, t)=\lim _{x=\nu \Delta x^{\prime}, \Delta x^{\prime} \rightarrow 0} v_{\nu}\left(t ; \Delta x^{\prime}\right) .
$$

It also implies the entropy condition: taking the limit $\Delta x \rightarrow 0$ in (3.9), it follows that $v_{\text {left }}>v_{\text {right }}$ across discontinuities. Thus,

$$
v(x, t)=\lim _{x=\nu \Delta x, \Delta x \rightarrow 0} v_{\nu}(t, \Delta x)
$$

is the unique, physically relevant solution of (1.2). The desired convergence of the LF scheme is, of course, well-known. The point made here was to show that by virtue of the one-sided Lipschitz condition (3.9), one can deduce both the entropysatisfying convergence as well as quantitative insight into the large-time behavior of the numerical solution.

4. Time-Decay in the Genuinely Nonlinear LF Scheme (cont'd.). In this section we continue our discussion on the time-decay of the LF scheme

$$
v_{\nu}(t+k)=\frac{v_{\nu+1}(t)+v_{\nu-1}(t)}{2}-\frac{\lambda}{2}\left(f_{\nu+1}-f_{\nu-1}\right)
$$


in the genuinely nonlinear case, say, strictly convex case, $\dot{a}_{*}=\operatorname{Min}_{v} \dot{a}(v)>0$, restricted by the weaker CFL-like condition $\lambda \sup _{\nu}\left|\Delta f_{\nu} / \Delta v_{\nu}\right| \leqslant 1$, due to monotonicity preserving.

THEOREM 4.1. Let $d, d>1$, be arbitrary, and consider the LF scheme (4.1) under the CFL-like condition

$$
\lambda \sup _{\nu}\left|\frac{\tilde{\Delta} f_{\nu}}{\tilde{\Delta} v_{\nu}}\right| \leqslant \frac{t+k(d-1)}{t+k(d+1)} .
$$

Then (3.3) holds, i.e.,

$$
\tilde{D}(t) \leqslant \operatorname{Max}\left[\frac{2}{(t+d k) \dot{a}_{*}}, \frac{d k}{t+d k} \tilde{D}(t=0)\right] .
$$

Proof. We repeat the induction step used in the proof of Theorem 3.1, establishing (3.4). Differencing (4.1) we find, as before,

$$
\tilde{\Delta} v_{\nu}(t+k)=\frac{\tilde{\Delta} v_{\nu+1}(t)+\tilde{\Delta} v_{\nu-1}(t)}{2}-\frac{\lambda}{2}\left(\tilde{\Delta} f_{\nu+1}-\tilde{\Delta} f_{\nu-1}\right) \text {. }
$$

By the mean-value theorem

$$
\tilde{\Delta} f_{\nu}=a\left(\bar{v}_{\nu}\right) \tilde{\Delta} v_{\nu}, \quad a\left(\bar{v}_{\nu}\right)=\int_{\theta=0}^{1} a\left(v_{\nu}(\theta)\right) d \theta, \quad v_{\nu}(\theta)=v_{\nu}+\theta \tilde{\Delta} v_{\nu} .
$$

Inserting (4.5) into (4.4), one obtains

$$
\tilde{\Delta} v_{\nu}(t+k)=\frac{1+\lambda a\left(\bar{v}_{\nu-1}\right)}{2} \tilde{\Delta} v_{\nu-1}(t)+\frac{1-\lambda a\left(\bar{v}_{\nu+1}\right)}{2} \tilde{\Delta} v_{\nu+1}(t),
$$

or, after division by $2 \Delta x$,

$$
\tilde{D}_{\nu}(t+k)=\frac{1+\lambda a\left(\bar{v}_{\nu-1}\right)}{2} \tilde{D}_{\nu-1}(t)+\frac{1-\lambda a\left(\bar{v}_{\nu+1}\right)}{2} \tilde{D}_{\nu+1}(t) .
$$

We distinguish between three cases:

(i) The shock-rarefaction interaction case. Assume $\tilde{D}_{\nu-1}(t) \cdot \tilde{D}_{\nu+1}(t)<0$. If $\tilde{D}_{\nu+1}(t)$ $>0$, then $\tilde{D}_{\nu-1}(t)<0$ and from $(4.6 \mathrm{~b})$

$$
\tilde{D}_{\nu}(t+k) \leqslant \frac{1-\lambda a\left(\bar{v}_{\nu+1}\right)}{2} \tilde{D}(t)
$$

while if $\tilde{D}_{\nu-1}(t)>0$, then $\tilde{D}_{\nu+1}(t)<0$ and from $(4.6 \mathrm{~b})$,

$$
\tilde{D}_{\nu}(t+k) \leqslant \frac{1+\lambda a\left(\bar{v}_{\nu-1}\right)}{2} \tilde{D}(t) \text {. }
$$

In either case, (4.2) implies

$$
\begin{aligned}
\tilde{D}_{\nu}(t+k) & \leqslant \frac{1+\lambda\left|a\left(\bar{v}_{\nu \pm 1}\right)\right|}{2} \tilde{D}(t) \\
& \leqslant \frac{1}{2}\left(1+\frac{t+k(d-1)}{t+k(d+1)}\right) \frac{d \lambda}{t+d k} M=\frac{d \lambda}{t+k+d k} M .
\end{aligned}
$$

(ii) The shock case. $\tilde{D}_{\nu-1}(t), \tilde{D}_{\nu+1}(t) \leqslant 0$. Then by $(4.6 \mathrm{~b})$

$$
\tilde{D}_{\nu}(t+k) \leqslant 0<\frac{d \lambda}{t+k+d k} M .
$$


(iii) The rarefaction case. Assume $\tilde{D}_{\nu-1}(t), \tilde{D}_{\nu+1}(t) \geqslant 0$. Our purpose is to reproduce the recursive inequality (3.8) which in turn led us to the desired bound (4.3). We rewrite with the help of (4.5)

$$
\begin{aligned}
\tilde{\Delta} f_{\nu+1}-\tilde{\Delta} f_{\nu-1} & =a\left(\bar{v}_{\nu+1}\right) \tilde{\Delta} v_{\nu+1}-a\left(\bar{v}_{\nu-1}\right) \tilde{\Delta} v_{\nu-1} \\
& =a\left(\bar{v}_{\nu+1}\right)\left(\tilde{\Delta} v_{\nu+1}-\tilde{\Delta} v_{\nu-1}\right)+\left(a\left(\bar{v}_{\nu+1}\right)-a\left(\bar{v}_{\nu-1}\right)\right) \tilde{\Delta} v_{\nu-1},
\end{aligned}
$$

where, using the mean-value theorem once more, we find

$$
\begin{aligned}
& a\left(\bar{v}_{\nu+1}\right)-a\left(\bar{v}_{\nu-1}\right)=\int_{\theta=0}^{1}\left[a\left(v_{\nu+1}(\theta)\right)-a\left(v_{\nu-1}(\theta)\right)\right] d \theta \\
& =\int_{\theta=0}^{1} \int_{\eta=0}^{1} \dot{a}\left[\eta v_{\nu+1}(\theta)+(1-\eta) v_{\nu-1}(\theta)\right] d \eta\left[(1-\theta) \tilde{\Delta} v_{\nu-1}+\theta \tilde{\Delta} v_{\nu+1}\right] d \theta .
\end{aligned}
$$

Inserting (4.8) into (4.7) and (4.7) into (4.4) we obtain, after division by $2 \Delta x$,

$$
\begin{aligned}
\tilde{D}_{\nu}(t+k)= & \frac{1+\lambda a\left(\bar{v}_{\nu+1}\right)}{2} \tilde{D}_{\nu-1}(t)+\frac{1-\lambda a\left(\bar{v}_{\nu+1}\right)}{2} \tilde{D}_{\nu+1}(t) \\
& -k \int_{\theta=0}^{1} \int_{\eta=0}^{1} \dot{a}[\cdots] d \eta(1-\theta) d \theta \tilde{D}_{\nu-1}^{2}(t) \\
& -k \int_{\theta=0}^{1} \int_{\eta=0}^{1} \dot{a}[\cdots] d \eta \theta d \theta \tilde{D}_{\nu-1}(t) \tilde{D}_{\nu+1}(t) .
\end{aligned}
$$

Similarly, if we rewrite with the help of (4.5)

$$
\tilde{\Delta} f_{\nu+1}-\tilde{\Delta} f_{\nu-1}=a\left(\bar{v}_{\nu-1}\right)\left(\tilde{\Delta} v_{\nu+1}-\tilde{\Delta} v_{\nu-1}\right)+\left(a\left(\bar{v}_{\nu+1}\right)-a\left(\bar{v}_{\nu-1}\right)\right) \tilde{\Delta} v_{\nu+1}
$$

and express the difference inside the second parentheses on the right in terms of (4.8), substitution into (4.4) gives us after division by $2 \Delta x$

$$
\begin{aligned}
\tilde{D}_{\nu}(t+k)= & \frac{1+\lambda a\left(\bar{v}_{\nu-1}\right)}{2} \tilde{D}_{\nu-1}(t)+\frac{1-\lambda a\left(\bar{v}_{\nu-1}\right)}{2} \tilde{D}_{\nu+1}(t) \\
& -k \int_{\theta=0}^{1} \int_{\eta=0}^{1} \dot{a}[\cdots] d \eta \theta d \theta \tilde{D}_{\nu+1}^{2}(t) \\
& -k \int_{\theta=0}^{1} \int_{\eta=0}^{1} \dot{a}[\cdots] d \eta(1-\theta) d \theta \tilde{D}_{\nu-1}(t) \tilde{D}_{\nu+1}(t) .
\end{aligned}
$$

Since according to our assumption $\tilde{D}_{\nu-1}(t), \tilde{D}_{\nu+1}(t) \geqslant 0$, the fourth terms on the RHS of (4.9a) and (4.9b) are nonnegative, while the sum of their first two terms does not exceed $\operatorname{Max}\left[\tilde{D}_{\nu-1}(t), \tilde{D}_{\nu+1}(t)\right]$; we therefore find

$$
\begin{aligned}
& \tilde{D}_{\nu}(t+k) \leqslant \operatorname{Max}\left[\tilde{D}_{\nu-1}(t), \tilde{D}_{\nu+1}(t)\right]-k \int_{\theta=0}^{1} \int_{\eta=0}^{1} \dot{a}[\cdots] d \eta(1-\theta) d \theta \tilde{D}_{\nu-1}^{2}(t), \\
& \tilde{D}_{\nu}(t+k) \leqslant \operatorname{Max}\left[\tilde{D}_{\nu-1}(t), \tilde{D}_{\nu+1}(t)\right]-k \int_{\theta=0}^{1} \int_{\eta=0}^{1} \dot{a}[\cdots] d \eta \theta d \theta \tilde{D}_{\nu+1}^{2}(t) .
\end{aligned}
$$

Since $\dot{a}[\cdots] \geqslant \dot{a}_{*}$, each of the double integrals on the RHS of the last two inequalities is greater than $\frac{1}{2} \dot{a}_{*}$, implying

$$
\begin{aligned}
& \tilde{D}_{\nu}(t+k) \leqslant \operatorname{Max}\left[\tilde{D}_{\nu-1}(t), \tilde{D}_{\nu+1}(t)\right]-\frac{k \dot{a}_{*}}{2} \tilde{D}_{\nu-1}^{2}(t), \\
& \tilde{D}_{\nu}(t+k) \leqslant \operatorname{Max}\left[\tilde{D}_{\nu-1}(t), \tilde{D}_{\nu+1}(t)\right]-\frac{k \dot{a}_{*}}{2} \tilde{D}_{\nu+1}^{2}(t) .
\end{aligned}
$$

The last two inequalities amount to (3.8) and the proof is completed as before. 
The one-sided Lipschitz bound appearing on the RHS of (4.3) is the maximum between the two terms

$$
\frac{2}{(t+d k) \dot{a}_{*}}, \quad \frac{d k}{t+d k} \tilde{D}(t=0)
$$

each of which involves a free parameter $d, d>1$. For $d \gg 1$, the first term becomes negligible and is dominated by the second $\tilde{D}(t=0)$ - the initial strength of rarefaction. With $d \geq 1$, on the other hand, the first term dominates, provided no strong rarefactions are present. This is certainly the case after quite some time, when possible strong initial rarefaction is dissolved. Observe that as $d$ decreases, we are forced to use a stricter CFL limitation

$$
\lambda \sup _{\nu}\left|\frac{\tilde{\Delta} f_{\nu}}{\tilde{\Delta} v_{\nu}}\right| \leqslant \frac{t+k(d-1)}{t+k(d+1)},
$$

so that possible strong rarefaction will be dissipated. Thus, there are essentially two situations:

(i) There exist no strong initial rarefactions, $\tilde{D}(t=0)=O(1)$ (in particular, with smooth initial data). Then we can take $d \gg 1$, easing the CFL limitation and giving inverse linear time-decay due to the first term $\sim t^{-1}$.

(ii) Strong initial rarefactions are present, $\tilde{D}(t=0)=O\left(h^{-1}\right)$. Then we should choose $d \gtrsim 1$ with CFL limitation $\lambda \sup _{\nu}\left|\tilde{\Delta} f_{\nu} / \tilde{\Delta} v_{\nu}\right| \leqslant t /(t+2 k)$, which over a time period $T \sim \lambda$ (i.e., after $O\left(h^{-1}\right)$ time steps) will cause these strong rarefactions to dissipate. Afterwards, we are back in the first situation where no strong rarefactions are present, and again, there is a time-decay $\sim t^{-1}$.

It is clear that by using the time-uniform CFL-like requirement

$$
\lambda \sup _{\nu}\left|\frac{\tilde{\Delta} f_{\nu}}{\tilde{\Delta} v_{\nu}}\right| \leqslant 1-\varepsilon, \quad \varepsilon>0,
$$

we get the same inverse linear time-decay; indeed, taking $d>2 / \varepsilon-1$ will do for the above analysis. It shows that taking the full CFL limitation, $\varepsilon \sim 0$, corresponds to taking large $d$ which may delay dissolving the rarefactions if initially strong-only after $n \sim \varepsilon^{-1}$ time-steps will the strong rarefaction fully dissipate, allowing the inverse linear time-decay to dominate.

Note, in particular, that as $d \rightarrow \infty$, allowing the extreme CFL-like requirement $\lambda \sup _{\nu}\left|\tilde{\Delta} f_{v} / \tilde{\Delta} v_{v}\right|=1$ to be used is not enough to dissolve strong rarefactions if present, despite the scheme being monotonicity-preserving in this case; the estimate (4.3) reduces to $\tilde{D}(t) \leqslant \tilde{D}(t=0)$, and the following example bears out its sharpness.

Example 4.2. Consider the LF scheme (4.1) applied to Burgers' equation, $f(u)=u^{2}$, with initial data $v_{\nu}(t=0)=\delta_{\nu 0}$ and $\lambda=1$. The extreme CFL-like requirement $\lambda \sup _{\nu}\left|\tilde{\Delta} f_{\nu} / \tilde{\Delta} v_{\nu}\right|=1$ is trivially satisfied, yet the initial, physically irrelevant spike will travel one mesh to the right at the time, without being dissolved. The LF scheme amounts, in this case, to a pure translation, lacking the dissipation to cause any decay.

The above example does not contradict the convergence of the LF scheme to the physically relevant limit solution due to its monotonicity, as much as it shows, as 
was mentioned above, the importance of using the stronger CFL limitation $\lambda \sup _{v}|a(v)| \leqslant 1$ - the correct one to guarantee monotonicity.

\section{ICASE}

NASA Langley Research Center

Hampton, Virginia 23665

1. M. Crandall \& A. Majda, "Monotone difference approximations for scalar conservation laws," Math. Comp., v. 34, 1980, pp. 1-21.

2. B. ENGQuist \& S. OSHER, "One-sided difference approximations for nonlinear conservation laws," Math. Comp., v. 36, 1981, pp. 321-351.

3. J. Guimm, "Solutions in the large for nonlinear hyperbolic systems of equations," Comm. Pure Appl. Math., v. 18, 1965, pp. 697-715.

4. S. K. GodUNov, "A finite difference method for the numerical computation of discontinuous solutions of the equations of fluid dynamics," Mat. Sb., v. 47, 1959, pp. 271-290.

5. A. HARTEN, The Method of Artificial Compression: I. Shocks and Contact Discontinuities, AEC Research \& Development Report C00-3077-50, Courant Institute, New York University, June 1974.

6. A. Harten, "High resolution schemes for hyperbolic conservation laws," J. Comput. Phys., v. 49, 1983, pp. 357-393.

7. A. Harten, J. M. Hyman \& P. D. Lax, "On finite difference approximations and entropy conditions for shocks," Comm. Pure Appl. Math., v. 29, 1976, pp. 297-302.

8. D. Hoff, "A constructive theory for shock-free, isentropic flow," J. Differential Equations, v. 38, 1980, pp. 1-31.

9. P. D. LAX, "Development of singularities of solutions of nonlinear hyperbolic partial differential equations," J. Math. Phys., v. 5, 1964, pp. 611-613.

10. P. D. Lax, Hyperbolic Systems of Conservation Laws and the Mathematical Theory of Shock Waves, SIAM Regional Conference Lectures in Applied Mathematics, No. 11, 1972.

11. J. P. LiU \& C. H. WANG, On a Hyperbolic System of Conservation Laws Which Is Not Strictly Hyperbolic, University of Wisconsin-Madison, MRC Report No. 2184, 1980.

12. E. M. MURMan, "Analysis of embedded shock waves calculated by relaxation methods," AIAA J., v. 12,1974 , pp. 626-633.

13. S. OSHER, "Riemann solvers, the entropy condition, and difference approximation," SIAM J. Numer. Anal., v. 21, 1984, pp. 217-235. 